\title{
Astragalin inhibits autophagy-associated airway epithelial fibrosis
}

\author{
In-Hee Cho ${ }^{\dagger}$, Yean-Jung Choi ${ }^{\dagger}$, Ju-Hyun Gong, Daekeun Shin, Min-Kyung Kang and Young-Hee Kang ${ }^{*}$
}

\begin{abstract}
Background: Fibrotic remodeling of airway and lung parenchymal compartments is attributed to pulmonary dysfunction with an involvement of reactive oxygen species (ROS) in chronic lung diseases such as idiopathic pulmonary fibrosis and asthma.

Methods: The in vitro study elucidated inhibitory effects of astragalin, kaempferol-3-O-glucoside from leaves of persimmon and green tea seeds, on oxidative stress-induced airway fibrosis. The in vivo study explored the demoting effects of astragalin on epithelial to mesenchymal transition in BALB/C mice sensitized with ovalbumin (OVA).

Results: The exposure of $20 \mu \mathrm{M} \mathrm{H}_{2} \mathrm{O}_{2}$ for $72 \mathrm{~h}$ accelerated E-cadherin loss and vimentin induction in airway epithelial BEAS-2B cells, which was reversed by non-toxic astragalin at 1-20 $\mu \mathrm{M}$. Astragalin allayed the airway tissue levels of ROS and vimentin enhanced by OVA challenge. Collagen type 1 production increased in $\mathrm{H}_{2} \mathrm{O}_{2}$-exposed epithelial cells and collagen fiber deposition was observed in OVA-challenged mouse airways. This study further investigated that the oxidative stress-triggered autophagic regulation was responsible for inducing airway fibrosis. $\mathrm{H}_{2} \mathrm{O}_{2}$ highly enhanced the expression induction of the autophagy-related beclin-1 and light chains $3 A / B$ (LC3A/B) within $4 \mathrm{~h}$ and astragalin blocked such induction by $\mathrm{H}_{2} \mathrm{O}_{2}$. This compound deterred the ROS-promoted autophagosome formation in BEAS-2B cells. Consistently, in OVA-sensitized mice the expression of beclin-1 and LC3A/B was highly induced, and oral administration of astragalin suppressed the autophagosome formation with inhibiting the induction of these proteins in OVA-challenged airway subepithelium. Induction of autophagy by spermidine influenced the epithelial induction of E-cadherin and vimentin that was blocked by treating astragalin.
\end{abstract}

Conclusion: These results demonstrate that astragalin can be effective in allaying ROS-promoted bronchial fibrosis through inhibiting autophagosome formation in airways.

Keywords: Astragalin, Autophagy, Epithelial to mesenchymal transition, Oxidative stress, Pulmonary fibrosis

\section{Introduction}

Autophagy, a catabolic process, plays a role in the elimination of damaged organelles and protein aggregates and in the turnover of essential proteins $[1,2]$. This process is activated by a diverse array of cellular stressors such as endoplasmic reticulum (ER) stress and microbial infection $[3,4]$. Especially, the autophagic pathways are modulated by oxidative stress response to oxygen tension and oxidant [5]. There is an emerging body of evidence that autophagy regulates various cellular processes and cell fates, including cellular death and senescence, inflammation and immune

\footnotetext{
* Correspondence: yhkang@hallym.ac.kr

${ }^{\dagger}$ Equal contributors

Department of Food and Nutrition, Hallym University, Chuncheon, Kangwon-do 200-702, Korea
}

function [6]. In addition, maladaptive or pathogenic aftereffects such as aberrant accumulation of proteins are observed. Accordingly, impaired autophagy has been implicated in many pathological conditions of neurological disorders, inflammatory bowel disease, diabetes and cancer [7]. Targeting autophagic pathways and their regulatory components may lead to the development of therapeutics.

Some environmental factors such as air pollutants may cause an extreme increase of reactive oxygen species (ROS) generation in the airways [8]. Oxidative stress contributes to airway and lung damage and consequently to several respiratory inflammatory diseases, including cystic fibrosis and chronic obstructive pulmonary disease [9]. Also, excessive production of ROS 
is thought to play a pivotal role in the pathogenesis of asthma, where exhaled levels of ROS positively correlate with disease severity [10]. Recent investigation has shown a crucial involvement of autophagy in the pathogenic processes underlying pulmonary diseases [11]. Indeed, autophagy is observed in the lung in response to oxidative stress evoked by the exposure to environmental toxicants $[7,11]$. Thus, the role of autophagy in pulmonary toxicity may offer new clues to strategies to treat lung injury linked to oxidative stress [12]. However, whether autophagy is responsible for promoting cell survival or cytotoxicity in the airways and lung remains elusive. In addition, the function of autophagy in the disease pathogenesis is still unclear and may entail impaired or elevated autophagic activity or imbalances in the activation of autophagic proteins.

Asthma is associated with a cytokine milieu that promotes transforming growth factor- $\beta 1$ (TGF- $\beta 1$ )-affiliated airway remodeling and loss of lung function [10]. TGF- $\beta 1$, a pleiotropic cytokine that has been established as a central mediator of tissue fibrosis, regulates autophagy and manipulates many critical aspects of disease-conditions associated with fibrosis and injury responses [13]. Hence, understanding the cellular mechanism of TGF- $\beta 1$-related autophagic process is crucial for identifying potential new therapeutic targets. On the other hand, a recent report indicates that fibroblasts in idiopathic pulmonary fibrosis acquire resistance to apoptosis and autophagy [14]. Autophagy inhibition induces acceleration of epithelial cell senescence and myofibroblast differentiation of lung fibroblasts which is an underlying mechanism of the pathogenesis of idiopathic pulmonary fibrosis [15].

Astragalin (Figure 1A), kaempferol-3-O-glucoside from leaves of persimmon and green tea seeds, possesses antiinflammatory activity [16,17]. One investigation reveals that pretreatment with astragalin can improve survival during lethal endotoxemia and attenuate inflammatory responses in a murine model of lipopolysaccharide (LPS)-induced acute lung injury [17]. Our recent study showed that astragalin antagonized endotoxin-induced oxidative stress leading to epithelial eosinophilia and

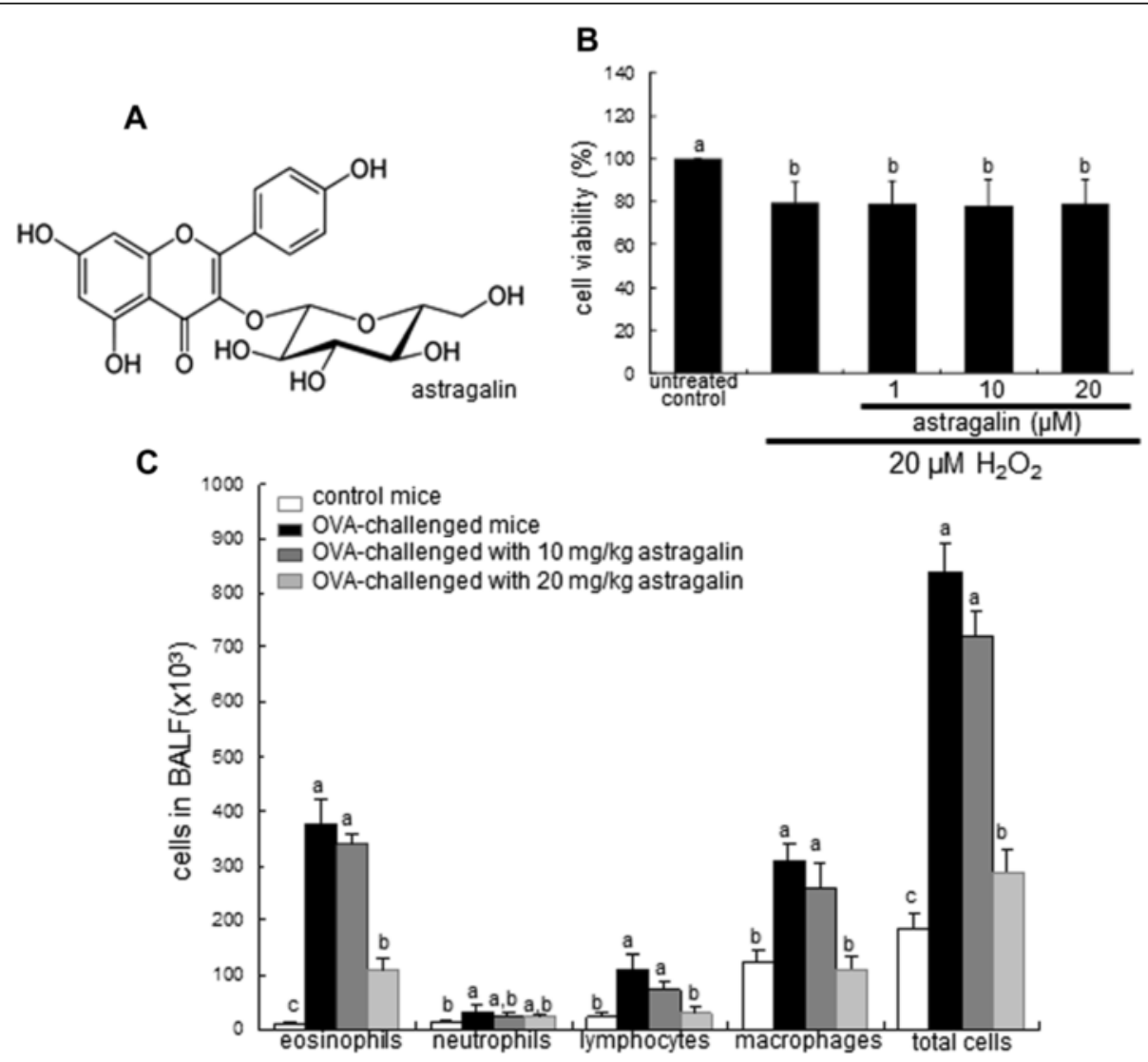

Figure 1 Chemical structure of astragalin (A), cytoprotection of astragalin against $\mathrm{H}_{2} \mathrm{O}_{2}$ (B), and number of neutrophils, eosinophils and basophils in the bronchoalveolar lavage fluid (C, BALF). BEAS-2B cells were cultured with $20 \mu \mathrm{M} \mathrm{H}_{2} \mathrm{O}_{2}$ in the absence and presence of 1-20 $\mu \mathrm{M}$ astragalin for $72 \mathrm{~h}(\mathrm{~B})$ and cell viability (mean $\pm \mathrm{SEM}, \mathrm{n}=4$ ) was measured by MTT assay. BALF was harvested from BALB/C mice $(n=6$, each group) $24 \mathrm{~h}$ after the final challenge with OVA or PBS vehicle (C). The bar graphs represent quantitative results and means without a common letter differ, $P<0.05$. 
apoptosis [18]. However, the inhibitory effects of astragalin on pulmonary fibrosis and autophagy are not yet elucidated. Based on the literature evidence that astragalin possesses antioxidant property and anti-allergic activity $[17,18]$, the current study investigated whether astragalin inhibited epithelial to mesenchymal transition (EMT) leading to pulmonary fibrosis and autophagic activity of $\mathrm{H}_{2} \mathrm{O}_{2}$-exposed airway epithelial BEAS-2B cells and in ovalbumin (OVA)-challenged mice. Furthermore, the interconnection between pulmonary fibrosis and autophagy under oxidative episodes was explored to emphasize a role underlying autophagy in asthmatic fibrosis.

\section{Materials and Methods Chemicals}

M199, human epidermal growth factor (EGF), hydrocortisone, gelatin, human insulin, apotransferrin and $\mathrm{H}_{2} \mathrm{O}_{2}$ were obtained from the Sigma-Aldrich Chemical (St. Louis, MO), as were all other reagents, unless specifically stated elsewhere. Fetal bovine serum (FBS), penicillin-streptomycin and trypsin-EDTA were purchased from the Lonza (Walkersville, MD). The human bronchial airway epithelial cell line BEAS-2B cells were provided by the American Type Culture Collection (Manassas, VA). Chicken egg white albumin was purchased from the Sigma-Aldrich Chemical and Imject Alum obtained from the Thermo Fisher Scienctific (Rodkford, IL). Human collagen type-1 antibody was purchased from the Santa Cruz Biotechnology (Santa Cruz, CA). Human E-cadherin antibody was provided by Cell Signaling Technology (Beverly, MA). Antibodies of human beclin-1 and microtubule-associated protein 1 light chain 3A/B (LC3A/B) were obtained from Abcam (Cambridge, UK). Horseradish peroxidaseconjugated goat anti-rabbit IgG, donkey anti-goat IgG and goat anti-mouse IgG were purchased from Jackson Immuno-Research Laboratories (West Grove, PA). Albumin from bovine serum (essentially fatty acid freeBSA) and skim milk were supplied by Becton Dickinson Company (Sparks, MD). 4',6-Diamidino-2-phenylindole (DAPI) was obtained from Santa Cruz Biotechnology and monodansylcadaverine (MDC) was purchased from Sigma-Aldrich Chemical. Astragalin was dissolved in dimethyl sulfoxide (DMSO) for live culture with cells; a final culture concentration of DMSO was $<0.5 \%$.

\section{BEAS-2B cell culture and viability}

The human lung epithelial BEAS-2B cells were cultured in 25 mM HEPES-buffered M199 containing 10\% FBS, $2 \mathrm{mM}$ L-glutamine, $100 \mathrm{U} / \mathrm{ml}$ penicillin, $100 \mu \mathrm{g} /$ $\mathrm{ml}$ streptomycin supplemented with $2.5 \mu \mathrm{g} / \mathrm{ml}$ insulin, $361 \mathrm{ng} / \mathrm{ml}$ hydrocortisone, $2.5 \mu \mathrm{g} / \mathrm{ml}$ apotransferrin and $20 \mathrm{ng} / \mathrm{ml} \mathrm{EGF}$. BEAS-2B cells were sustained in
90-95\% confluence at $37^{\circ} \mathrm{C}$ in an atmosphere of $5 \%$ $\mathrm{CO}_{2}$. In order to induce airway fibrosis, BEAS-2B cells were pretreated with 1-20 $\mu \mathrm{M}$ astragalin and then stimulated with $20 \mu \mathrm{M} \mathrm{H}_{2} \mathrm{O}_{2}$ for up to $72 \mathrm{~h}$. Non$\mathrm{H}_{2} \mathrm{O}_{2}$-treated cells were also incubated under the same conditions as those used for the $\mathrm{H}_{2} \mathrm{O}_{2}$ protocols. Astragalin did not improve the epithelial injury due to $20 \mu \mathrm{M} \mathrm{H}_{2} \mathrm{O}_{2}$ (Figure 1B).

\section{Western blot analysis}

Whole BEAS-2B cell lysates were prepared in $1 \mathrm{mM}$ Tris- $\mathrm{HCl}$ (pH 6.8) lysis buffer containing $10 \%$ SDS, $1 \%$ glycerophosphate, $0.1 \mathrm{mM} \mathrm{Na}_{3} \mathrm{VO}_{4}, 0.5 \mathrm{mM} \mathrm{NaF}$ and protease inhibitor cocktail. Cell lysates containing equal amounts of proteins and equal volume of culture media were electrophoresed on 6-15\% SDS-PAGE and transferred onto a nitrocellulose membrane. Blocking a non-specific binding was performed using either $3 \%$ fatty acid-free BSA or $5 \%$ non-fat dry milk for $3 \mathrm{~h}$. The membrane was incubated overnight at $4^{\circ} \mathrm{C}$ with a specific primary antibody of E-cadherin, vimentin, collagen type-1, beclin-1 and LC3A/B. The membrane was then applied to a secondary antibody conjugated to horseradish peroxidase for $1 \mathrm{~h}$. Following another triple washing, the target protein was determined using the Immobilon Western Chemiluminescent HRP Substrate (Millipore Corp., Billerica, MA) and the Agfa medical X-ray film blue (Agfa HealthCare NV, Mortsel, Belgium). Incubation with mouse anti-human $\beta$-actin antibody was conducted for the comparative control.

\section{Murine asthma models}

Six week-old male BALB/c mice (Hallym University Breeding Center for Laboratory Animals) were used in the present study. Mice were kept on a $12 \mathrm{~h}$ light $/ 12 \mathrm{~h}$ dark cycle at $23 \pm 1{ }^{\circ} \mathrm{C}$ with $50 \pm 10 \%$ relative humidity under specific pathogen-free conditions, fed a nonpurified diet (RodFeed ${ }^{\mathrm{TM}}$, DBL, Umsung, Korea) and were provided with water ad libitum at the animal facility of Hallym University. The non-purified diet composition is as follows: NLT (Not Less Than) 20.5\% crude protein, NLT 3.5\% crude fat, NMT (Not More Than) $8.0 \%$ crude fiber, NMT $8.0 \%$ crude ash, NLT $0.5 \%$ calcium and NLT $0.5 \%$ phosphorus. Mice were allowed to acclimatize for 1 week before beginning the experiments. Mice were divided into four subgroups ( $\mathrm{n}=6 \mathrm{for}$ each subgroup). Mice were sensitized with $20 \mu \mathrm{g}$ OVA dissolved in a solution of $30 \mu \mathrm{l}$ PBS and $50 \mu \mathrm{l}$ Imject Alum by a subcutaneous injection twice on day 0 and day 14. For the dietary interventions, $0.1 \mathrm{ml}$ astragalin solution (10 or $20 \mathrm{mg} / \mathrm{kg} \mathrm{BW}$ ) was orally administrated to OVA-sensitized mice $1 \mathrm{~h}$ before challenge. On day 28, 29 and 30, 5\% OVA inhalation to mice was performed for $20 \mathrm{~min}$ in a plastic chamber linked to an 
ultrasonic nebulizer (Clenny ${ }^{2}$ Aerosol, Medel, Italy). Control mice were sensitized and challenged with PBS as the OVA vehicle. Twenty four hours after the latest provocation (day 30), all mice were killed with an anesthetic $(2 \mu \mathrm{l} / \mathrm{kg}$ rompun and $8 \mu \mathrm{l} / \mathrm{kg}$ zoletil, i.p.). The trachea was cannulated, and both lungs and airways were rinsed in $1 \mathrm{ml}$ PBS for the collection of bronchoalveolar lavage fluid (BALF). The numbers of inflammatory cells including eosinophils in BALF were determined using a Hemavet HV950 Multispecies Hematologic Analyzer (Drew Scientific, Oxford, CT). The right lungs were collected, frozen in liquid nitrogen and kept at $-80^{\circ} \mathrm{C}$ until used for Western blotting. Left lungs were preserved and fixed in $4 \%$ paraformaldehyde, and then used for immunohistochemical analyses. The total inflammatory cell number in the BALF of mice exposed to OVA increased with a predominance of eosinophils (Figure 1C). The oral administration of astragalin deterred the increase in the number of eosinophils in OVA-treated mice, indicating that astragalin alleviated airway inflammation.

The present study was approved by the Hallym University Institutional Review Board and Committee on Animal Experimentation (hallym R2014-6). All experiments were performed in compliance with the University's Guidelines for the Care and Use of Laboratory Animals. No mice were dead and no apparent signs of exhaustion were observed during the experimental period.

\section{Measurement of ROS production}

The lung tissue production of ROS was measured using 2'-7'-dichlorofluorescein diacetate (DCF-DA) that is hydrolyzed and oxidized by ROS to a fluorescent compound 2'-7'-DCF. After lysation and incubation of lung tissue extracts with DCF-DA for $30 \mathrm{~min}$, the fluorescence intensity was read in a Fluoroskan reader (Thermo Fisher Scientific, Waltham, MA) with an appropriate filter.

\section{Immunohistochemical staining}

For the immunofluorescent histochemical analysis, paraffin-embedded lung tissue sections $(5 \mu \mathrm{m}$ thickness) were deparaffinized and hydrated. The sections were pre-incubated in a boiling sodium citrate buffer (10 mM sodium citrate, $0.05 \%$ Tween $20, \mathrm{pH}$ 6.0) for antigen retrieval. The tissues were blocked with $5 \%$ BSA in PBS for $1 \mathrm{~h}$. Specific primary antibody against mouse vimentin, mouse beclin-1, mouse LC3A/B or mouse $\alpha$-smooth muscle actin ( $\alpha$-SMA) was incubated with the tissue sections overnight. Subsequently, the tissue sections were incubated for $1 \mathrm{~h}$ with FITC-conjugated anti-goat IgG or Cy3-conjugated anti-goat IgG. For identification of nuclei, the fluorescent nucleic acid dye
DAPI was applied for $10 \mathrm{~min}$. Stained tissues were mounted on slides using fluorescent mounting medium (Vector Laboratories, Burlingame, CA). Images of each slide were taken using an optical microscope Axioimager system (Zeiss, Gottingen, Germany).

\section{Masson trichrome staining}

For the histological analyses, lung specimens obtained at the end of the experiments were fixed in $10 \%$ buffered paraformaldehyde. The paraffin-embedded lung specimen were sectioned at $5 \mu \mathrm{m}$ thickness, deparaffinized and stained with Masson trichrome for the light microscopic visualization of collagen fibers and muscle fibers. The stained tissue sections were examined using an optical microscope system, and five images were taken for each section.

\section{MDC staining}

Labeling of autophagic vacuoles was carried out by using the autofluorescent compound MDC. BEAS-2B cells were treated with $20 \mu \mathrm{M} \mathrm{H}_{2} \mathrm{O}_{2}$ to in the presence of 1-20 $\mu \mathrm{M}$ astragalin for $4 \mathrm{~h}$ on a glass-chamber slide and were incubated in MDC for $30 \mathrm{~min}$. Following the incubation, cells were washed three times with PBS and immediately photographed with a fluorescence microscope.

The formation of autophagosomes in lung tissues was measured by using lung specimen fixed in $10 \%$ buffered paraformaldehyde. The paraffin-embedded lung tissue sections were deparaffinized and stained with MDC for the microscopic visualization. The fluorescent DAPI was applied for the nuclear counterstaining.

\section{Statistical analysis}

The results were expressed as mean \pm SEM for each treatment group in each experiment. Statistical analyses were performed using Statistical Analysis Systems statistical software package (SAS Institute, Cary, NC). Significance was determined by one-way analysis of variance, followed by Duncan range test for multiple comparisons. Differences were considered significant at $P<0.05$.

\section{Results}

\section{Inhibition of airway EMT and fibrosis by astragalin}

The loss of the epithelial marker E-cadherin and the gain of the mesenchymal marker vimentin are fundamental events in EMT and important changes in the process of fibrosis [19]. BEAS-2B cells exposed to $20 \mu \mathrm{M} \mathrm{H}_{2} \mathrm{O}_{2}$ up to $72 \mathrm{~h}$ induced a change from epithelial phenotype to mesenchymal (Figure 2A). The expression of E-cadherin was reduced at $48 \mathrm{~h}$ following the $\mathrm{H}_{2} \mathrm{O}_{2}$ stimulation of BEAS-2B cells. When 1-20 $\mu \mathrm{M}$ astragalin was added to epithelial cells in the presence of $\mathrm{H}_{2} \mathrm{O}_{2}$, the cellular 

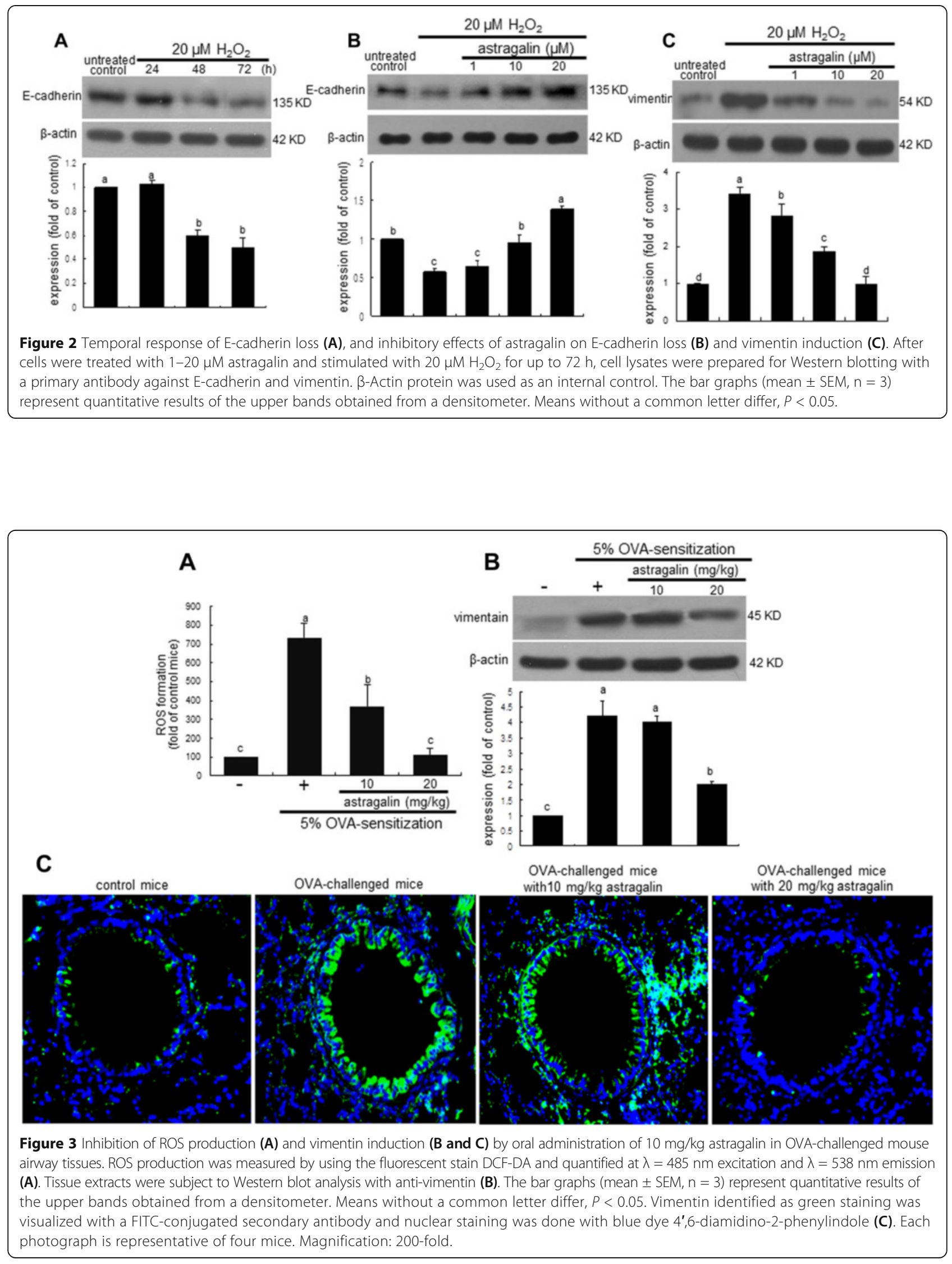
expression of E-cadherin was restored (Figure 2B). On the other hand, the $72 \mathrm{~h}$ stimulation of $\mathrm{H}_{2} \mathrm{O}_{2}$ markedly enhanced the epithelial expression of vimentin (Figure 2C). When 1-20 $\mu \mathrm{M}$ astragalin was supplemented to $\mathrm{H}_{2} \mathrm{O}_{2}$-exposed BEAS-2B cells, the vimentin induction was dosedependently downregulated. Accordingly, oxidative stress may instigate EMT process and fibrosis in airways, which can be disturbed by supplementing astragalin to the epithelium.
This study attempted to determine whether asthmatic injury triggered airway fibrosis, which was dampened by the supplementation of astragalin. The OVA challenge enhanced the ROS production in airway tissues and such elevation was dose-dependently diminished by administrating $10-20 \mathrm{mg} / \mathrm{kg}$ astragalin (Figure 3A). Vimentin is commonly used as a marker of cells of mesenchymal origin. Western blot analysis revealed that epithelial expression of vimentin typical in EMT was promoted by the

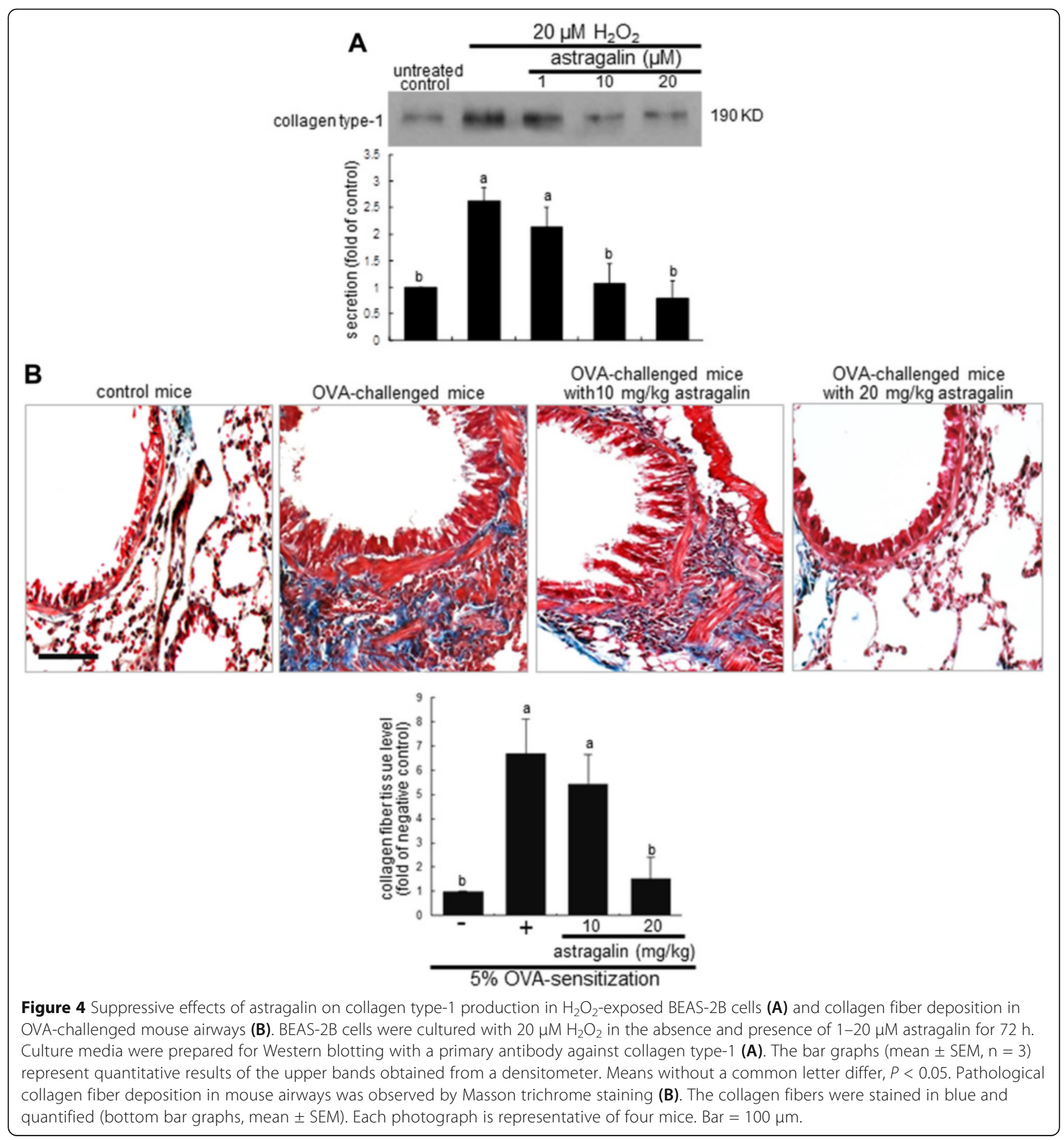


OVA challenge (Figure 3B). However, the oral administration of $20 \mathrm{mg} / \mathrm{kg}$ astragalin diminished the elevated induction of vimentin in airway tissues by the OVA episode. In addition, the FITC-green staining of vimentin disappeared in the airway epithelium of $20 \mathrm{mg} / \mathrm{kg}$ astragalin-exposed mice (Figure 3C).

Subepithelial fibrosis in the airways entails the increased deposition extracellular matrix (ECM) such as fibrogenic collagen by myofibroblasts and submucosal resident fibroblasts and fibrocytes expressing profibrotic cytokines [20]. The epithelial production of collagen type-I was elevated in BEAS-2B cells exposed to $\mathrm{H}_{2} \mathrm{O}_{2}$ and such production was suppressed by adding $\geq 10 \mu \mathrm{M}$ astragalin (Figure 4A). This study further investigated that astragalin ameliorated the structural remodeling of airways due to increased deposition of ECM components. The collagen fiber deposition was notably observed (blue color) in airway tissues of OVA-challenged mice, evidenced by Masson's trichrome staining (Figure 4B). There was a dense deposition of collagen fibers around the bronchial tissues of OVAchallenged mice. On the contrary, the oral treatment of OVA-sensitized mice with $10-20 \mathrm{mg} / \mathrm{kg}$ astragalin reduced the collagen fiber deposition and alleviated subepithelial fibrosis (Figure 4B). In addition, it should be noted that the OVA sensitization induced epithelial cell modulation and goblet cell hyperplasia (red color).

\section{Blockade of airway autophagy by astragalin}

When BEAS-2B cells were incubated with $20 \mu \mathrm{M} \mathrm{H}_{2} \mathrm{O}_{2}$ up to $24 \mathrm{~h}$, epithelial expression of beclin-1 was strikingly elevated at $4 \mathrm{~h}$ after $\mathrm{H}_{2} \mathrm{O}_{2}$ injury and thereafter diminished (Figure 5A). However, its $4 \mathrm{~h}$-epithelial induction was encumbered by treating cells with $\geq 10 \mu \mathrm{M}$ astragalin (Figure 5B). Consistently, the OVA inhalation to mice led to the increased levels of the autophagic marker beclin-1 in mouse airway tissues, evidenced by

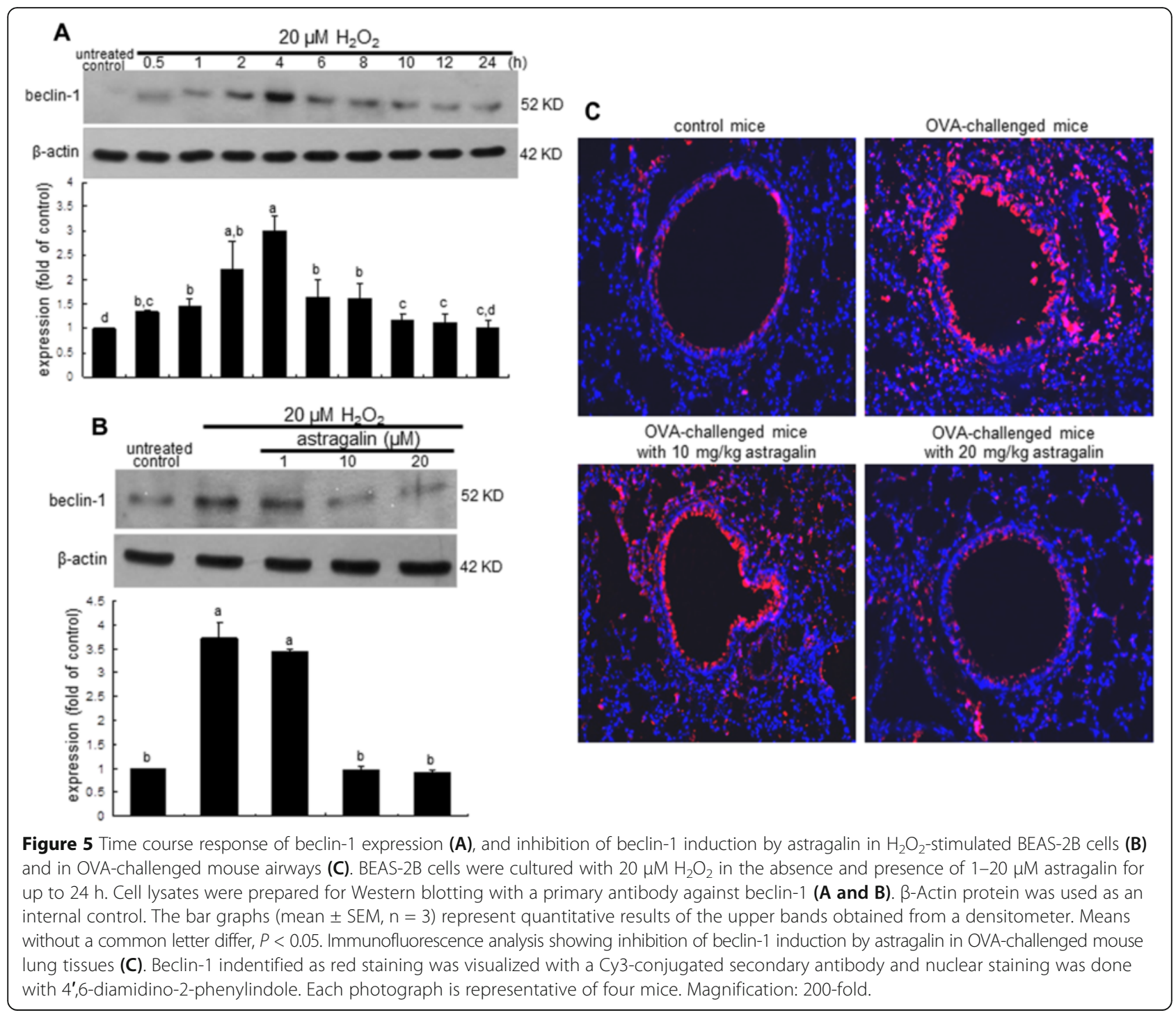


Cy3-red staining (Figure 5C). In contrast, the oral administration of $20 \mathrm{mg} / \mathrm{kg}$ astragalin allayed the beclin-1 induction (loss of pinkish staining) in OVA-challenged mouse airways. Microtubule-associated LC3A/B was highly enhanced at 2-4 h after treating $20 \mu \mathrm{M} \mathrm{H}_{2} \mathrm{O}_{2}$ to BEAS-2B cells (Figure 6A). The epithelial induction of LC3A/B by $\mathrm{H}_{2} \mathrm{O}_{2}$ was diminished in $\geq 10 \mu \mathrm{M}$ astragalin-treated BEAS2B cells exposed to $\mathrm{H}_{2} \mathrm{O}_{2}$ for $4 \mathrm{~h}$ (Figure 6B). Similarly, Western blot data showed that the airway tissue levels of LC3A/B increased in OVA-challenged mice, which was dampened by supplementing $\geq 10 \mathrm{mg} / \mathrm{kg}$ astragalin to the mice (Figure 6C).
Autophagy is characterized by the formation of doublemembrane autophagosomes that fuse with lysosomes to form autolysosomes [21]. This study attempted to confirm whether oxidative stress induced autophagosome formation in BEAS-2B cells. There was a strong MDC staining observed in the cytoplasts of BEAS-2B cells exposed to 20 $\mu \mathrm{M} \mathrm{H} \mathrm{H}_{2} \mathrm{O}_{2}$ (Figure 7A). When 1-20 $\mu \mathrm{M}$ astragalin was added to the cells, dose-dependent decreases in the number of autophagosomes and in the MDC staining intensity were observed (Figure 7A). Thus, astragalin may be an antagonist to the autophagy induction of BEAS-2B cells in response to $\mathrm{H}_{2} \mathrm{O}_{2}$. Autophagosome formation was further

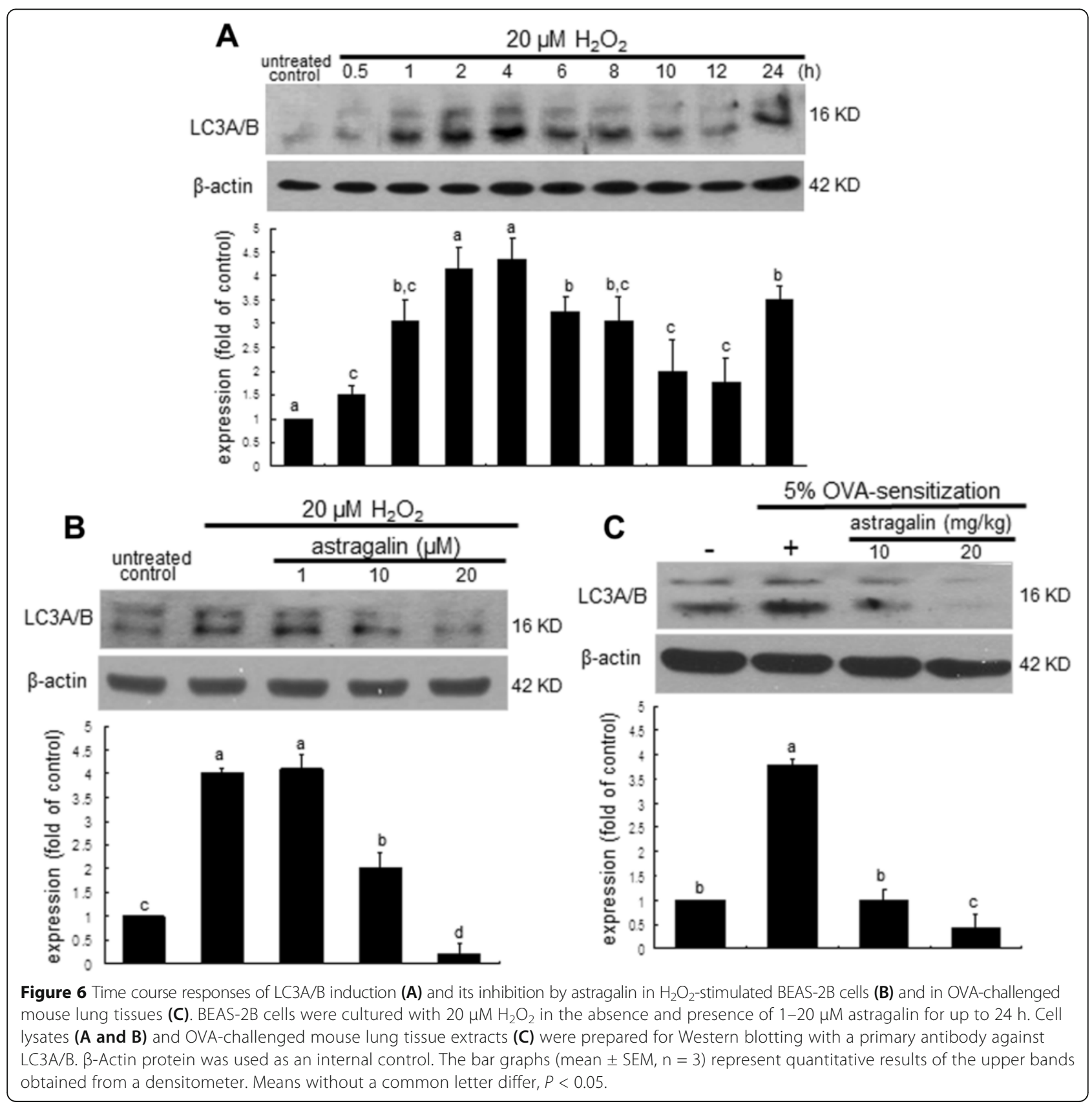




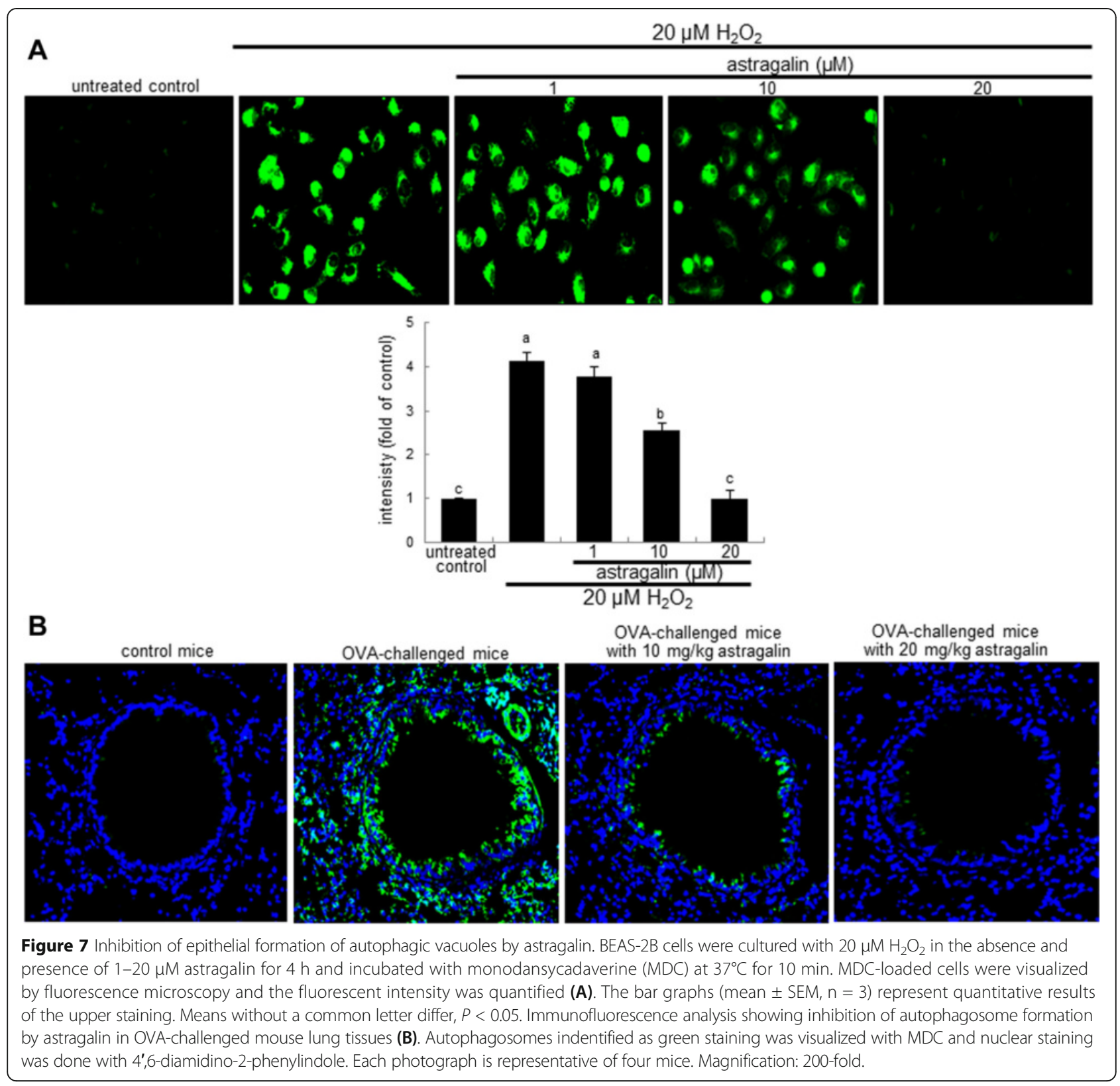

determined in airway tissues of OVA-challenged mice (Figure 7B). There was a strong MDC staining in cells around the airway subepithelium of OVA-inhaled mice. The autophagosomes were formed in similar regions of airways to those of the collagen fiber deposition (Figure 4B). When $10-20 \mathrm{mg} / \mathrm{kg}$ astragalin was orally treated to OVA-sensitized mice, the MDC staining was highly diminished (Figure 7B).

\section{Interconnection between airway fibrosis and autophagy}

The epithelial induction of both $\alpha$-SMA and LC3A/B highly increased in airway subepithelium of OVAchallenged mice (Figure 8A). Oral treatment of mice with $\geq 10 \mathrm{mg} / \mathrm{kg}$ astragalin allayed the induction of these proteins by OVA challenge. It should be noted that the induction of these proteins concurrently occurred in similar regions of airway tissues. This study attempted to reveal that epithelial autophagy might be involved in the cellular alteration of airway EMT process and fibrosis. Oxidative stress due to $\mathrm{H}_{2} \mathrm{O}_{2}$ caused the induction of beclin-1 involved in the autophagosome formation (Figures $5 \mathrm{~A}$ and $8 \mathrm{~B}$ ). In addition, the polyamine spermidine highly increased epithelial beclin1 induction regardless of $\mathrm{H}_{2} \mathrm{O}_{2}$ presence (Figure 8B). The beclin-1 inducer promoted the vimentin induction and the E-cadherin loss. Accordingly, epithelial autophagy may be responsible for the EMT process in airways leading to fibrosis. 


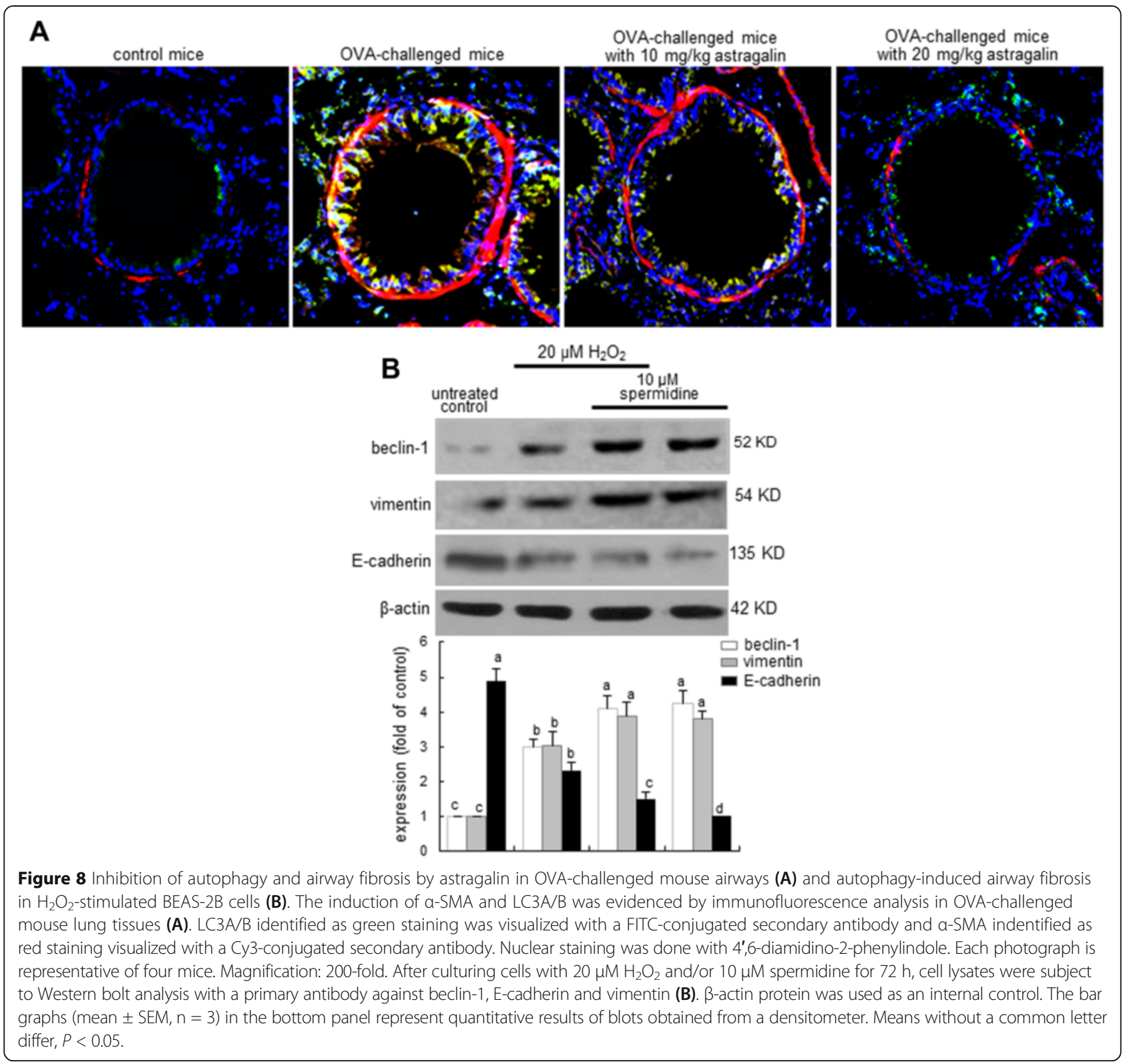

\section{Discussion}

Eight major findings were extracted from this study. 1) Astragalin reduced the number of eosinophils in the BALF of OVA-challenged BALB/c mice, indicating that its administration attenuated airway inflammation entailing eosinophil infiltration in peribronchial and perivascular airway. 2) BEAS-2B cells exposed to $20 \mu \mathrm{M} \mathrm{H}_{2} \mathrm{O}_{2}$ induced EMT process through reducing epithelial expression of E-cadherin and enhancing epithelial induction of vimentin. 3) When $1-20 \mu \mathrm{M}$ astragalin was added to epithelial cells in the presence of $\mathrm{H}_{2} \mathrm{O}_{2}$, the cellular expression of E-cadherin was restored, but the vimentin induction was dose-dependently attenuated. 4) Oral administration of astragalin encumbered epithelial cell excrescence and thickening by diminishing the vimentin induction and collagen fiber deposition in OVA-challenged mouse airway. 5) $\mathrm{H}_{2} \mathrm{O}_{2}$ markedly promoted epithelial induction of beclin-1 and LC3A/B within $4 \mathrm{~h}$ with increasing the autophagosome formation, which was reversed by astragalin. 6) When $\geq 10$ $\mathrm{mg} / \mathrm{kg}$ astragalin was treated to OVA-challenged mice, this compound suppressed the induction of beclin-1 and LC3A/B in airway tissues. 7) The protein induction of vimentin, $\alpha$-SMA and LC3A/B concurrently occurred in similar regions of airway tissues of OVAinhaled mice. 8) The autophagy inducer promoted the E-cadherin loss and enhanced the vimentin induction, indicating that autophagy appeared to be responsible for 
the airway EMT. Therefore, astragalin can be effective in deterring airway EMT and fibrosis through modulating oxidative stress-responsive signaling pathway linked to autophagy.

Oxidative stress causes airway and lung damage consequently leading to several respiratory inflammatory diseases such as chronic obstructive pulmonary disease $[9,10]$. In addition, allergic asthma due to the exposure to environmental antigens may entail an increase in endogenous ROS formation, causing oxidative stressinduced injury to the respiratory system and antioxidant defenses [22,23]. In this study the ROS production was highly induced in airway tissues of OVA-challenged mice. Recent research suggests that the use of redoxbased therapy to attenuate levels of ROS provides a potential strategy to alleviate oxidative stress-induced airway inflammation in patients with asthma [22,24]. Therapeutic interventions that reduce the exposure to environmental ROS or augment endogenous antioxidant defenses might be beneficial as adjunctive therapies in asthmatic patients [25]. Our recent study showed that astragalin may be a potent antioxidant antagonizing endotoxin-induced oxidative stress [18]. In addition, astragalin blunted $\mathrm{H}_{2} \mathrm{O}_{2}$-promoted epithelial apoptosis concomitant with nuclear condensation or caspase- 3 activation. Currently, astragalin suppressed the airway infiltration of inflammatory cells following the exposure of mice to the OVA antigen.

Although the specific mechanisms responsible for chronic obstructive pulmonary diseases are still being unraveled, ROS radicals are important mediators of airway tissue damage. Oxidative stress has been implicated as an important molecular mechanism underlying fibrosis in the lungs [26]. However, the causal role of ROS released from inflammatory/interstitial cells in mediating fibrosis is not firmly established. Pulmonary fibrosis, a progressive and lethal lung disease, is characterized by inflammation and accumulation of ECM components [27]. This study showed that the oxidant $\mathrm{H}_{2} \mathrm{O}_{2}$ enhanced the collagen type- 1 production and the OVA antigen augmented the collagen fiber deposition in the airway subepithelial compartments. Pulmonary fibrosis as well as how best to relieve an imbalance in ROS production would be major therapeutic challenges for which new strategies are warranted. In the present study astragalin as an antioxidant was effective in encumbering airway fibrosis triggered by oxidative stress. Unfortunately, this study did not examine the key origins of oxidative stress in OVA-induced pulmonary fibrosis. Nevertheless, novel insights into unique therapeutic targets of astragalin for managing ROS-induced pulmonary fibrosis need to be provided.

ROS and markers of oxidative stress play fundamental roles in the pathology of idiopathic pulmonary fibrosis characterized by interstitial fibrosis governing irreversible deformation of pulmonary architecture, possibly through the induction of EMT [28]. One investigation showed that the potent antioxidant $\mathrm{N}$-acetylcysteine inhibited TGF- $\beta 1$-induced EMT in a rat epithelial cell line and in primary rat alveolar epithelial cells, indicating that oxidative stress might be responsible for pulmonary function and the antioxidant possess its beneficial effects on idiopathic pulmonary fibrosis [29]. In this study astragalin allayed the EMT process by restoring Ecadherin expression and reducing vimentin induction in oxidant-experienced airway epithelial cells and OVAchallenged airway tissues. There is growing evidence suggesting that EMT entails TGF- $\beta 1$ in alveolar epithelial cells and may contribute to airway remodeling in severe asthma and fibrotic lung diseases [10]. In addition, the association between ROS and TGF- $\beta 1$-induced fibrosis has been discussed [26]. Oxidative stress elicits the production of cytokines and growth factors that may play a pivotal role in invasive myofibroblastic differentiation and collagen deposition. One can speculate that astragalin alleviated oxidative stress and blocked TGF- $\beta 1$ production, which might lead to the inhibition of airway EMT and fibrosis.

A recent study shows that TGF- $\beta 1$ regulates autophagy involved in fibrosis of kidney diseases including tubulointerstitial fibrosis, glomerulosclerosis, and diabetic nephropathy [13]. This study emphasized an emerging role of the autophagy in pulmonary fibrosis. The regulation of epithelial autophagy by TGF- $\beta 1$ can be implicated in the pathogenesis of asthmatic pulmonary fibrosis. Oxidative stress has been shown to be involved in the pathogenic process of autophagy $[7,10,12]$. In this study the autophagic stress caused by $\mathrm{H}_{2} \mathrm{O}_{2}$ accelerated the epithelial EMT, and the autophagic process concurrently occurred in airway fibrotic regions caused by OVA inhalation in mice. A recent insight into the cellular and molecular bases of the autophagic process will identify potential new therapeutic targets of pulmonary fibrosis.

Autophagy is required for hepatic fibrogenesis by activated hepatic stellate cells in mice and selective reduction of autophagic activity in fibrogenic cells might be used to treat patients with fibrotic diseases [30]. Consistently, the modulation of autophagy may have the potential to treat common respiratory ailments and disorders. In this study astragalin inhibited the autophagosome formation from oxidant-exposed airway epithelial cells and in airway tissues of OVA-inhaled mice through reducing the induction of beclin-1 and LC3A/B. These findings indicate that oral administration of astragalin may attenuate the oxidative stress-induced autophagy and consequent fibrosis. On the other hand, there are conflicting reports indicating that fibroblasts in idiopathic pulmonary fibrosis are resistant to 
apoptosis and autophagy concomitant with decreased expression of autophagic beclin-1 [14,31]. The phenolic compounds of rutin and curcumin function as a possible effective stimulator of fatty acid-induced autophagy via mTOR-dependent pathways in nonchemical inducedhepatic stellate cells that can transdifferentiate into myofibroblast-like cells, indicating their benefits for alleviating liver fibrosis [32]. In addition, the autophagy inhibition induces acceleration of epithelial cell senescence [15]. Accordingly, whether autophagy is responsible for promoting epithelial toxicity or survival in airways and lung should be clarified. Ultimately, the unique role of autophagy in pulmonary toxicity linked to oxidative stress may provide novel clues to strategies to treat pulmonary fibrosis [12].

\section{Conclusion}

This study investigated the potential of astragalin as a modulator antagonizing oxidative stress-associated autophagy leading to pulmonary fibrosis. Astragalin restored the expression of the epithelial marker E-cadherin and suppressed the $\mathrm{H}_{2} \mathrm{O}_{2}$ induction of the mesenchymal vimentin in epithelial cells and in OVA-challenged mouse airway tissues. Oral administration of astragalin inhibited subepithelial deposition of collagen fibers in OVA-sensitized mouse airways. In addition, astragalin reduced the bronchial subepithelial induction of the autophagic beclin-1 and LC3A/B enhanced by $\mathrm{H}_{2} \mathrm{O}_{2}$ oxidant and OVA antigen. Ultimately, astragalin encumbered the induction of EMT and fibrosis under the milieu of oxidative stress and autophagic injury in mouse airways. Therefore, astragalin was effective in ameliorating oxidative stress-associated pulmonary fibrosis through disturbing airway EMT and epithelial autophagic stress.

\section{Competing interests}

The authors (I-H. Cho, Y-J. Choi, J-H. Gong, D. Shin, M-K. Kang and Y-H. Kang) declare that they have no competing interests.

\section{Authors' contributions}

$I H C, J H G$ and YHK designed research; IHC, JHG and DS conducted research; IHC, JHG and MKK analyzed data; IHC, YJC and YHK wrote the paper; YHK had primary responsibility for final content. All authors read and approved the final manuscript.

\section{Acknowledgements \\ This study was supported by National Research Foundation of Korea (2012012946) and by the Ministry of Education, Science Technology and National Research Foundation of Korea through the Human Resource Training Project for Regional Innovation (2012-01-A-05-003-12-010100).}

Received: 5 December 2014 Accepted: 10 April 2015

Published online: 21 April 2015

\section{References}

1. Li Y, Zhang J, Chen X, Liu T, He W, Chen Y. Molecular machinery of autophagy and its implication in cancer. Am J Med Sci. 2012;343:155-61.

2. Choi AM, Ryter SW, Levine B. Autophagy in human health and disease. N Engl J Med. 2013;368:651-62.
3. Huang J, Lam GY, Brumell JH. Autophagy signaling through reactive oxygen species. Antioxid Redox Signal. 2011;14:2215-31.

4. Fung TS, Liu DX. Coronavirus infection, ER stress, apoptosis and innate immunity. Front Microbiol. 2014;5:296.

5. Navarro-Yepes J, Burns M, Anandhan A, Khalimonchuk O, del Razo LM, Quintanilla-Vega B, et al. Oxidative stress, redox signaling, and autophagy: cell death versus survival. Antioxid Redox Signal. 2014;21:66-85.

6. Ryter SW, Cloonan SM, Choi AM. Autophagy: a critical regulator of cellular metabolism and homeostasis. Mol Cells. 2013;36:7-16.

7. Kongara S, Karantza V. The interplay between autophagy and ROS in tumorigenesis. Front Oncol. 2012;2:171.

8. Zuo L, Otenbaker NP, Rose BA, Salisbury KS. Molecular mechanisms of reactive oxygen species-related pulmonary inflammation and asthma. Mol Immunol. 2013;56:57-63.

9. Lee IT, Yang CM. Role of NADPH oxidase/ROS in pro-inflammatory mediators-induced airway and pulmonary diseases. Biochem Pharmacol. 2012:84:581-90

10. Poon A, Eidelman D, Laprise C, Hamid Q. ATG5, autophagy and lung function in asthma. Autophagy. 2012;8:694-5.

11. Araya J, Hara H, Kuwano K. Autophagy in the pathogenesis of pulmonary disease. Intern Med. 2013;52:2295-303.

12. Malaviya R, Laskin JD, Laskin DL. Oxidative stress-induced autophagy: role in pulmonary toxicity. Toxicol Appl Pharmacol. 2014;275:145-51.

13. Ding $Y$, Choi ME. Regulation of autophagy by TGF- $\beta$ : emerging role in kidney fibrosis. Semin Nephrol. 2014;34:62-71.

14. Ricci A, Cherubini E, Scozzi D, Pietrangeli V, Tabbì L, Raffa S, et al. Decreased expression of autophagic beclin 1 protein in idiopathic pulmonary fibrosis fibroblasts. J Cell Physiol. 2013;228:1516-24.

15. Araya J, Kojima J, Takasaka N, Ito S, Fujii S, Hara H, et al. Insufficient autophagy in idiopathic pulmonary fibrosis. Am J Physiol Lung Cell Mol Physiol. 2013;304:L56-69.

16. Li F, Liang D, Yang Z, Wang T, Wang W, Song $X$, et al. Astragalin suppresses inflammatory responses via down-regulation of $\mathrm{NF}-\mathrm{kB}$ signaling pathway in lipopolysaccharide-induced mastitis in a murine model. Int Immunopharmacol. 2013;17:478-82.

17. Soromou LW, Chen N, Jiang L, Huo M, Wei M, Chu X, et al. Astragalin attenuates lipopolysaccharide-induced inflammatory responses by down-regulating NF-kB signaling pathway. Biochem Biophys Res Commun. 2012:419:256-61.

18. Cho IH, Gong JH, Kang MK, Lee EJ, Park JH, Park SJ, et al. Astragalin inhibits airway eotaxin-1 induction and epithelial apoptosis through modulating oxidative stress-responsive MAPK signaling. BMC Pulm Med. 2014;14:122.

19. Ponnusamy MP, Seshacharyulu P, Lakshmanan I, Vaz AP, Chugh S, Batra SK. Emerging role of mucins in epithelial to mesenchymal transition. Curr Cancer Drug Targets. 2013;13:945-56.

20. Yamauchi $\mathrm{K}$, Inoue $\mathrm{H}$. Airway remodeling in asthma and irreversible airflow limitation-ECM deposition in airway and possible therapy for remodeling. Allergol Int. 2007:56:321-9.

21. Macintosh RL, Ryan KM. Autophagy in tumour cell death. Semin Cancer Biol. 2013:23:344-51.

22. Jiang L, Diaz PT, Best TM, Stimpfl JN, He F, Zuo L. Molecular characterization of redox mechanisms in allergic asthma. Ann Allergy Asthma Immunol. 2014;113:137-42

23. Hoffman SM, Tully JE, Nolin JD, Lahue KG, Goldman DH, Daphtary N, et al. Endoplasmic reticulum stress mediates house dust mite-induced airway epithelial apoptosis and fibrosis. Respir Res. 2013;14:141.

24. Kirkham P, Rahman I. Oxidative stress in asthma and COPD: antioxidants as a therapeutic strategy. Pharmacol Ther. 2006;111:476-94.

25. Bowler RP. Oxidative stress in the pathogenesis of asthma. Curr Allergy Asthma Rep. 2004;4:116-22.

26. Cheresh P, Kim SJ, Tulasiram S, Kamp DW. Oxidative stress and pulmonary fibrosis. Biochim Biophys Acta. 1832;2013:1028-40.

27. Camelo A, Dunmore R, Sleeman MA, Clarke DL. The epithelium in idiopathic pulmonary fibrosis: breaking the barrier. Front Pharmacol. 2014;4:173.

28. Inghilleri S, Morbini P, Campo I, Zorzetto M, Oggionni T, Pozzi E, et al. Factors influencing oxidative imbalance in pulmonary fibrosis: an immunohistochemical study. Pulm Med. 2011;2011:421409.

29. Felton VM, Borok Z, Willis BC. N-acetylcysteine inhibits alveolar epithelialmesenchymal transition. Am J Physiol Lung Cell Mol Physiol. 2009;297:L805-12. 
30. Hernández-Gea V, Ghiassi-Nejad Z, Rozenfeld R, Gordon R, Fiel MI, Yue Z, et al. Autophagy releases lipid that promotes fibrogenesis by activated hepatic stellate cells in mice and in human tissues. Gastroenterology. 2012;142:938-46.

31. Patel AS, Lin L, Geyer A, Haspel JA, An CH, Cao J, et al. Autophagy in idiopathic pulmonary fibrosis. PLoS One. 2012;7, e41394.

32. Lee KW, Thiyagarajan V, Sie HW, Cheng MF, Tsai MJ, Chia YC, et al. Synergistic effect of natural compounds on the fatty acid-induced autophagy of activated hepatic stellate cells. J Nutr Biochem. 2014;25:903-13.

\section{Submit your next manuscript to BioMed Central} and take full advantage of:

- Convenient online submission

- Thorough peer review

- No space constraints or color figure charges

- Immediate publication on acceptance

- Inclusion in PubMed, CAS, Scopus and Google Scholar

- Research which is freely available for redistribution 